\title{
Three-dimensional particle focusing under viscoelastic flow based on dean-flow-coupled elasto-inertial effects
}

\author{
D. Yuan a, J. Zhang ${ }^{a}$, S. Yan ${ }^{a}$, C. Pan ${ }^{\mathrm{a}}$, G. Alici ${ }^{\mathrm{a}}$, N. T. Nguyen ${ }^{\mathrm{b}}$, W. H. Li ${ }^{* a}$ \\ ${ }^{\text {a }}$ School of Mechanical, Materials and Mechatronic Engineering, University of \\ Wollongong, Wollongong, NSW, Australia 2522; ${ }^{\mathrm{b}}$ Queensland Micro and \\ Nanotechnology Centre, Griffith University, Brisbane QLD, Australia 4111
}

\begin{abstract}
Based on dean-flow-coupled elasto-inertial effects, 3D particle focusing in a straight channel with asymmetrical expansion-contraction cavity arrays (ECCA channel) is achieved. First, the mechanism of particle focusing in both Newtonian and non-Newtonian fluids was introduced. Then particle focusing was demonstrated experimentally in this channel with Newtonian and non-Newtonian fluids using three different sized particles $(3.2 \mu \mathrm{m}, 4.8 \mu \mathrm{m}, 13 \mu \mathrm{m})$, respectively. The influences of flow rates on focusing performance in ECCA channel were studied. Results show that in ECCA channel particles are focused on the cavity side in Newtonian fluid due to the synthesis effects of inertial and dean-drag force, whereas on the opposite cavity side in non-Newtonian fluid due to the addition of viscoelastic force. Compared with the focusing performance in Newtonian fluid, the particles are more easily and better focused in nonNewtonian fluid. A further advantage is three-dimensional (3D) particle focusing in non-Newtonian fluid is realized according to the lateral side view of the channel while only two-dimensional (2D) particle focusing can be achieved in Newtonian fluid. Conclusively, this Dean-flow-coupled elasto-inertial microfluidic device could offer a continuous, sheathless, and high throughput $\left(>10000 \mathrm{~s}^{-1}\right)$ 3D focusing performance, which may be valuable in various applications from high speed flow cytometry to cell counting, sorting, and analysis.
\end{abstract}

Keywords: Microfluidic, Dean-flow-coupled elasto-inertial effects, 3D particle focusing

\section{INTRODUCTION}

Three-dimensional (3D) particle focusing is essential for separating ${ }^{1}$, sorting, counting ${ }^{2}$, detecting and analysis in numerous biological and chemical applications, such as the flow cytometers used for the detection and enumeration of bio-particles.

The manipulation of particles is based on their intrinsic physical characteristics such as particle size, shape, density, polarizability and magnetic susceptibility. Focusing techniques can be classified to two categories: active methods and passive methods. Active methods are based on the application of external force fields, such as dielectrophoresis (DEP) $)^{3,4,5,6}$, magnetophoresis ${ }^{7,8}$, surface acoustic wave (SAW)-induced streaming ${ }^{9}$, and optical tweezers ${ }^{10}$. Passive methods are based on the microchannel geometrical effects and hydrodynamic forces ${ }^{11}$, such as pinched flow fractionation (PFF) ${ }^{12}$, hydrodynamic filtration ${ }^{13}$, Dean-flow coupled inertial effects ${ }^{14}{ }^{15}$, deterministic lateral displacement (DLD) ${ }^{16}$.

Most of particle focusing methods are performed in Newtonian fluid. Active methods can provide precise control of target bio-particles, however, they have a low throughput and require extra, expensive device components for the external forces. Passive methods are relatively simple, effective and have a high throughput, but some of the channel design are still very complex and lack flexibility. Moreover, either the active or the passive methods are difficult to realize 3D focusing in Newtonian fluid, however, in non-Newtonian fluids, with the aid of elasto-inertial effects, 3D sheathless particle focusing can be easily achieved in a simple square or rectangular channel by properly adjusting the flow rate. Recently, particle manipulation including focusing and separation have gained significant attention in nonNewtonian fluids. The positive first normal stress difference $\left(N_{l}\right)$ arising in pressure driven flows of dilute polymer solutions can lead suspended particles or cells to migrate to the mid-plane of the channel ${ }^{17,18,19,20}$. Leshansky, et al ${ }^{19}$ observed that the particles migrate toward the centreline due to the imbalance in the first normal stress difference between the centreline and the walls in a slit channel. These particles are two-dimensionally focused under the conditions

- weihuali@uow.edu.au; phone: +61 24221 3490; fax: +61 242213238

Seventh International Symposium on Precision Mechanical Measurements, edited by Liandong Yu, Proc. of SPIE Vol. 9903, 99030K - () 2016 SPIE

CCC code: $0277-786 \times / 16 / \$ 18 \cdot$ doi: $10.1117 / 12.2211265$ 
of negligible inertia and dominant elasticity. However, in planar rectangular channels, the elasticity results in particle migration towards the centreline and the corners of the channel which corresponds to the low first normal stress regions, so this approach cannot be directly applied to $3 \mathrm{D}$ focusing. Yang, et $\mathrm{al}^{21}$. reported that the number of multiple equilibrium positions can be reduced to one equilibrium at the centreline by properly adjusting the flow rate due to the synergetic effect of inertia and viscoelasticity. By balancing the elastic and inertial forces, he demonstrated particle focusing on a flow centreline of a square microchannel by using a synergetic combination of elasticity and inertia of viscoelastic fluid flow. D'Avino, et $\mathrm{al}^{22}$. performed $3 \mathrm{D}$ numerical simulations and experiments to demonstrate particle focusing through viscoelasticity-induced migration in pressure-driven flows in simple cylindrical micropipes. Seo, et $\mathrm{al}^{23}$, investigated the elasto-migration of microparticles in a microscale pipe flow of viscoelastic fluids using a holographic technique, and evaluated the effects of blockage ratio, flow rate, and entry length on particle migration.

In this paper, 3D particle focusing in ECCA channel was demonstrated by exploiting the Dean-flow-coupled elastoinertial effects. By properly balancing the inertial force, viscoelastic force and Dean-drag force, 3D particle focusing in non-Newtonian fluid along the opposite side of cavities was achieved. Particle focusing was experimentally demonstrated in this channel under Newtonian and non-Newtonian fluids using three different sized particles $(3.2 \mu \mathrm{m}, 4.8$ $\mu \mathrm{m}, 13 \mu \mathrm{m})$, respectively. The influences of flow rate on focusing performance were also studied. The particles are focused on the cavity side in a Newtonian fluid by the synthesis of inertial and Dean-drag force, whereas on the opposite cavity side in a non-Newtonian fluid due to the addition of viscoelastic force. In ECCA channel, the flow rate that allows particle focusing is dozens of or even hundreds of microlitre per minute, which is much higher than that in a straight channel (several microlitres per minute) ${ }^{21}$, resulting in a higher throughtput $\left(>10000 \mathrm{~s}^{-1}\right)$. To the authors' best knowledge, the Dean-flow-coupled elasto-inertial focusing in such a device has not been explored yet. This Dean-flow-coupled elasto-inertial microfluidic device reported here could offer a continuous, sheathless, and high throughput $\left(>10000 \mathrm{~s}^{-1}\right)$ 3D focusing performance, which may be valuable in various applications from high speed flow cytometry to cell counting, sorting, and analysis.

\section{THEORETICAL BACKGROUND}

\section{A. Particle focusing in Newtonian and non-Newtonian fluids}

In Newtonian fluids, the shear gradient lift force and wall lift force are the two dominant forces to govern the particle migration, and an equilibrium position is created by the balancing the two lift forces. The sum of the two inertial lift forces, which is called the net inertial lift force, was first derived by Asmolov ${ }^{24}$, and later simplified by Di Carlo ${ }^{14}$ as following:

$$
\begin{aligned}
& F_{L}=\frac{\rho_{f} U_{m}^{2} a^{4}}{D_{h}^{2}} f_{L}\left(R_{c}, x_{c}\right) \\
& R_{C}=\frac{\rho_{f} U_{m} D_{h}}{\mu_{f}}=\frac{2 \rho_{f} Q}{\mu_{f}(w+h)}
\end{aligned}
$$

where $\rho_{f}, U_{m}$ and $\mu_{f}$ are the fluid density, mean velocity, and dynamic viscosity, respectively; $a$ is the spherical diameter of the particles; $D_{h}=2 w h /(w+h)$ is the hydraulic diameter for a rectangular channel with $w$ and $h$ the width and height of the channel cross section. The lift coefficient of net inertial lift force $f_{L}\left(R_{c}, x_{c}\right)$ is a function of the position of the particles within the cross section of channel $x_{c}$ and the channel Reynolds number $R_{C}{ }^{14}$.

Particle migration and focusing in non-Newtonian viscoelastic fluid is different with that in Newtonian fluids. In nonNewtonian viscoelastic fluid, the elastic force is determined by the intrinsic properties of the medium. The elastic effects of a non-Newtonian fluid in the channel flow can be characterized by $W_{i}$, which is defined as the ratio between two time constants:

$$
W_{i}=\frac{\lambda}{t_{f}}=\lambda \dot{\gamma}=\lambda \frac{2 v_{m}}{w}=\frac{2 \lambda Q}{h w^{2}}
$$

where $\lambda$ is the relaxation time of the fluid and $t_{f}$ is the characteristic time of the channel flow. The characteristic time is approximately equal to the inverse of the average (characteristic) shear rate, $\dot{\gamma}$, which is $2 V_{m} / w$, or $2 \lambda Q / h w^{2}$ in a rectangular channel. $V_{m}$ is the average velocity and $w$ and $h$ is the channel width and height, respectively.

Figure 1 is the simulation results of velocity magnitude in the cross section of a rectangular channel $100 \mu \mathrm{m} \times 50 \mu \mathrm{m}$ (Width $\times$ Height) using COMSOL. (As the shear viscosity in non-Newtonian fluid is considered constant, there is no difference between Newtonian and viscoelastic flows in the velocity distribution within a cross-section at the same flow rate $^{25,26}$.) The color bar represents the magnitude of the velocity. The magnitude of velocity increases from the bottom of 
the bar (dark blue) to the top of the bar (dark red). It can be seen from the simulation results that the maximum velocity occurs in the central part of the rectangular channel, and decreases gradually from the centre line to the channel wall, which validates that the velocity profile is a parabolic curve. Figure 2 is the simulation results of the shear rate, which is the differential of the flow velocity. The centreline and the corners of the channel are low shear rate regions, which also correspond to the low first normal stress $N_{1}$ regions regions and particles tend to migrate towards the channel centreline and corners under pure elastic effects. The elastic force $F_{E}$ can be assumed to stem from the imbalance in the distribution of $N_{1}$ over the size of the particle $\mathrm{e}^{20,27}$,

$$
F_{E} \sim a^{3} \nabla N_{1}=a^{3}\left(\nabla \tau_{x x}-\nabla \tau_{y y}\right)=2 a^{3}(1-\beta) W_{i} \nabla \dot{\gamma}^{2}
$$

where $a$ is the particle radius, $\beta$ is the ratio of the solvent to solution viscosity and $\nabla \dot{\gamma}$ is the non-dimensional local shear rate.

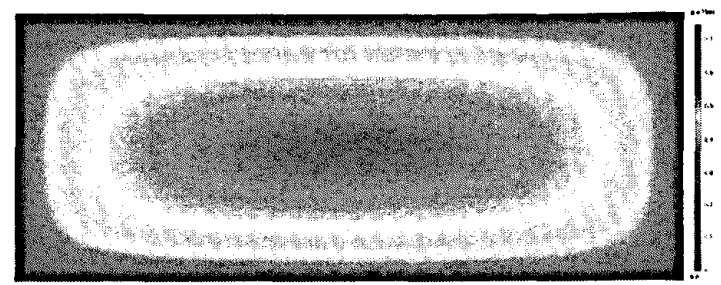

Figure 1 Simulation results of velocity magnitude.

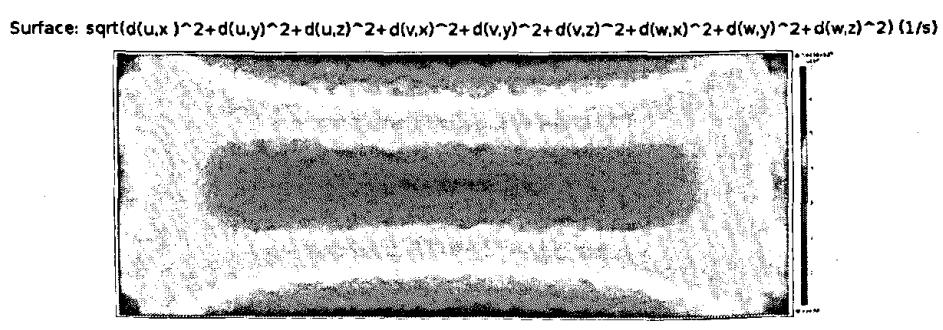

Figure 2 Simulation results of shear rate.

The number of multiple equilibrium positions can be reduced to one at the centreline by properly adjusting the flow rate due to the synergetic effect of inertia and viscoelasticity. The competition between the elasticity and the inertia determines the particle migration. Since the elasticity and inertia is represented by $W_{i}$ and $R c$, respectively, the elasticity number (the ratio between these two numbers $\left(W_{i} / R c\right)$ can be used to measure the relative importance of elastic forces to inertial effects, which is defined as:

$$
E l=\frac{w_{l}}{R_{C}}=\frac{\lambda \mu_{f}(w+h)}{\rho_{f} w^{2} h}
$$

If the medium does not yield apparent shear-thinning viscosity, $E l$ is independent of the flow rate and only dependent on fluid viscosity and relaxation time in a specific channel.

\section{B. Principle of Dean-flow-coupled elasto-inertial particle focusing}

In the ECCA channel with non-Newtonian fluid, three kinds of forces are harmonized: Lift force $F_{L}$, including the sheargradient lift force $\left(F_{L S}\right)$, wall-repulsion force $\left(F_{L W}\right)$; the Dean drag force $F_{D}$ resulting from the curved channel geometry; and elastic force $F_{E}$ induced by the nature of the viscoelastic medium. Figure 3 shows the simulation results of the flow field in the cross section $100 \mu \mathrm{m}$ away from the cavity along the $\mathrm{x}$ direction at the outlet in the ECCA channel using COMSOL and the schematic illustration of the focusing mechanism in Newtonian (a) and non-Newtonian (b) fluid. When particles flowing in the ECCA channel with a Newtonian fluid, the effects of inertial migration and secondary flow act in superposition on the particles, thus the four equilibrium positions induced by Lift force $F_{L}$ in rectangular channels are destroyed by the dean drag force $F_{D}$ and particles become stable in two modified equilibrium positions, Figure 3(a). The arrows represent the magnitude and direction of the secondary flow field induced by the abrupt contraction of the channel. When particles flowing in ECCA channels with a viscoelastic non-Newtonian fluid, the 
elastic force $F_{E}$ should be considered, which is directed away from the wall and decays with increasing distance from the wall. In a straight rectangular channel with non-Newtonian fluids, the particles tend to migrate to the centreline of the channel by the synergetic effect of inertia and viscoelasticity. In the ECCA channel, the particles become unstable when an additional secondary flow drag is exerted on the particles. Subsequently, a new equilibrium position can be achieved near the channel wall by the combined effects of lift force $F_{L}$, elastic force $F_{E}$ and the dean drag force $F_{D}$, as shown in Figure 3(b). Finally, 3D particle focusing is realized in an ECCA channel with the combined effect of Dean-flowcoupled elasto-inertial forces.
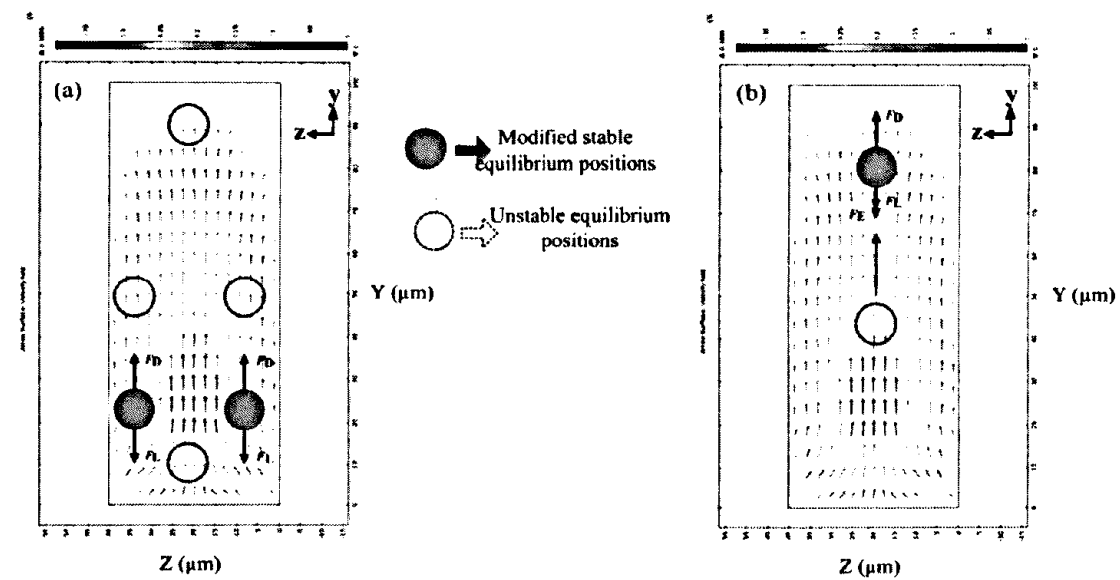

Figure 3 Simulation results of the flow field in the cross section $100 \mu \mathrm{m}$ away from the cavity along the $\mathrm{x}$ direction and the schematic illustration of the focusing mechanism in Newtonian (a) and non-Newtonian (b) fluid. (a) The red circles represent two equilibrium positions that resulted from a balance between the inertial lift force $F_{\mathrm{L}}$ and secondary flow drag $F_{\mathrm{D}}$. and $(b)$ The red circle represents the single equilibrium position by the synergetic effect of inertia, secondary flow and viscoelasticity. The dashed circles are the unstable equilibrium positions. The arrows represent the magnitude and direction of the secondary flow field induced by the abrupt contraction of the channel.

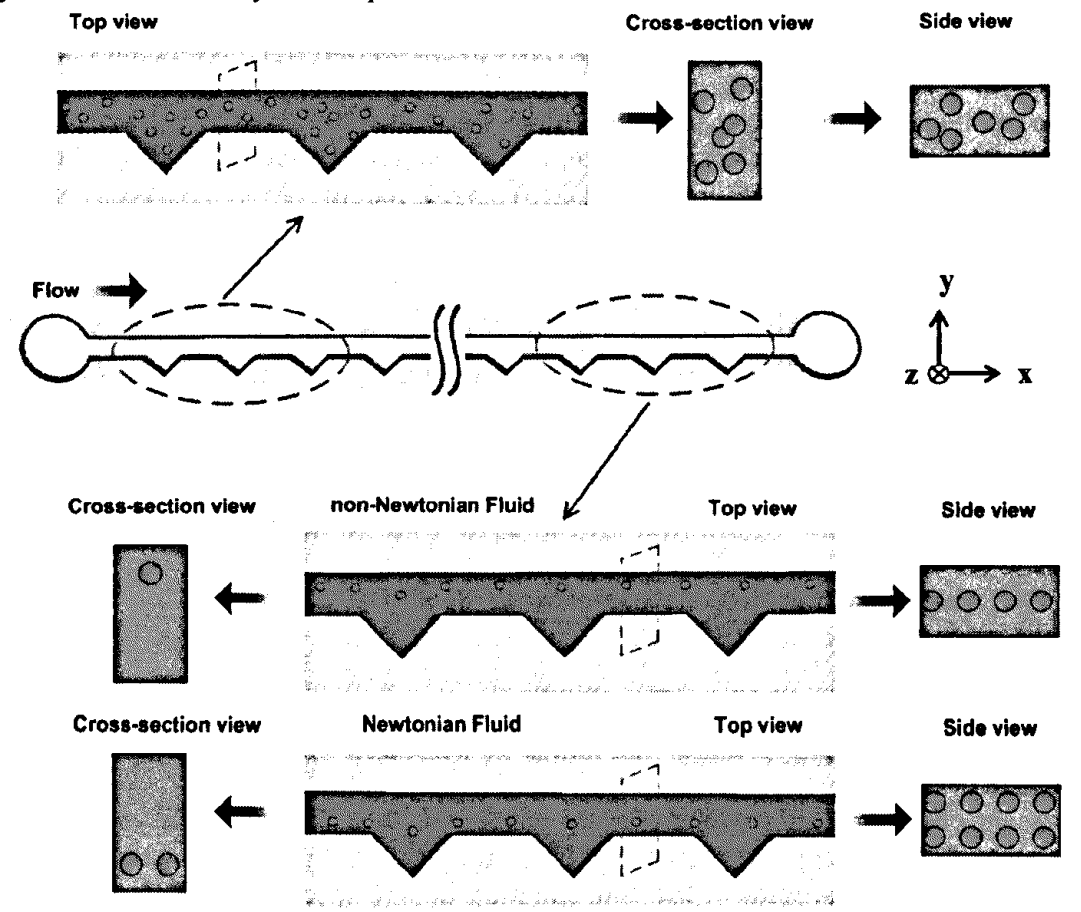

Figure 4 Schematic diagrams of particle focusing in Newtonian and non-Newtonian fluids in the ECCA channel. Particles are randomly injected to the inlet, and finally focused in a single line on the opposite cavity side at the outlet in nonNewtonian fluid in Dean-coupled elasto-inertial effects, while focused to two equilibrium positions on the cavity side at the outlet in Newtonian fluid in the combination of Dean and inertial effects. 
Figure 4 shows the schematic diagram of particle focusing in Newtonian and non-Newtonian fluids in the ECCA channel. There are 26 repeated expansion-contraction triangular cavities in this channel, while only the inlet and outlet section of the channel is illustrated here. Particles are randomly injected to the inlet, so from the top view, cross section view and side view in the amplified inlet section the particles are dispersed. As the particle suspension flows, particles are driven into a single line along the channel wall on the opposite cavity side in non-Newtonian fluid with the Dean-flow-coupled elasto-inertial effects described above. However, in a Newtonian fluid, because there is no elastic effect induced by the polymers, particles are focused to two equilibrium positions on the cavity side at the outlet in a Newtonian fluid as result of Dean and inertial effects.

\section{EXPERIMENTS}

\section{A. Design and fabrication of the microfluidic device}

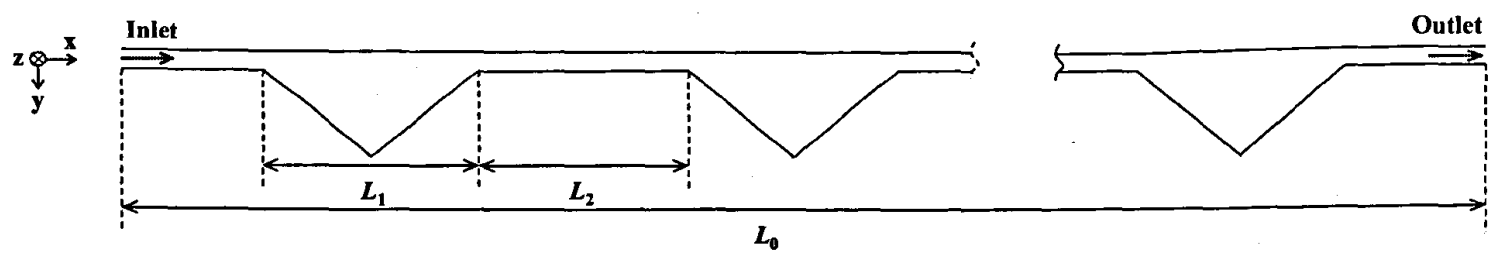

Figure 5 The schematic geometry of the microfluidic channel with triangular expansion-contraction cavity arrays, $L_{0}=48$ $\mathrm{mm}, L_{1}=900 \mu \mathrm{m}, L_{2}=900 \mu \mathrm{m}$.

The right angled isosceles triangular cavities are patterned on one side of a straight channel. The channel has a cross section of $100 \mu \mathrm{m} \times 40 \mu \mathrm{m}$ (width $\times$ height). The longest edge of the triangle is $L_{1}=900 \mu \mathrm{m}$, and the space between two adjacent cavities is uniform at $L_{2}=900 \mu \mathrm{m}$. The total length of the straight channel is $L_{0}=48 \mathrm{~mm}$, including 26 repeated expansion- contraction triangular cavities. Its schematic geometry is shown in Figure 5.

The device was fabricated by standard photolithography and soft lithography techniques. The fabrication included rapid prototyping on a silicon master, polydimethylsiloxane (PDMS) replica moulding, and sealing through plasma oxidation. Briefly, photoresist (SU-8 2025, MicroChem Co., Newton, MA) was spun on a silicon wafer at 2000 rpm to a thickness of $40 \mu \mathrm{m}$, and then exposed to UV light through a designed mask using a mask aligner system (ABM, San Jose, CA). After that the photoresist on the silicon wafer was developed in an SU-8 developer solution and rinsed by isopropylalcohol (IPA) to create a positive replica of channel geometry. A PDMS mixture with a 10:1 ratio of base to agent (Dow Corning, Midland, MI) was poured over the silicon master, degassed to remove bubbles in a vacuum oven, and cured at $100^{\circ} \mathrm{C}$ for $45 \mathrm{~min}$. After the PDMS was cured and taken out of silicon master, the inlet and outlet holes were punched with a custom needle tip. Finally, the PDMS slide was bonded with another PDMS slide after exposure to oxygen plasma (PDC-002, Harrick Plasma, Ossining, NY) for 3 minutes.

\section{B. Suspending fluid and particles}

In this work, two kinds of fluids were prepared: Newtonian fluid (deionized water), and Non-Newtonian fluid (moderate elasticity fluid). For the moderate elasticity fluid, PEO (poly (ethylene oxide), $\mathbf{M}_{\mathbf{w}}=2000000$, Sigma-Aldrich) was added to deionized water in $500 \mathrm{ppm}$. The density of the fluid matches with the polystyrene (PS) particles $\left(1.05 \mathrm{~g} \mathrm{~cm}^{3}\right)$. The PEO solution is considered to have a constant shear viscosity of $3.12 \times 10^{-3} \mathrm{~Pa} s$ under the present experimental conditions and its relaxation time is $9.1 \times 10^{-3} \mathrm{~s}$. The viscosities for deionized water is $1.0 \times 10^{-3} \mathrm{~Pa} \mathrm{~s}$. Tween $20(0.01 \mathrm{wt} \%$, Sigma-Aldrich) was added to all the solutions to prevent particle-particle adhesion.

Internally dyed fluorescent polystyrene microspheres were purchased from Thermo Fisher Scientific., USA. A particle suspension was prepared by diluting $3.2 \mu \mathrm{m}$ (product no.G0300, CV $<5 \%$ ), $4.8 \mu \mathrm{m}$ (product no.G0500, CV $<5 \%$ ), and 13 $\mu \mathrm{m}$ (product no.G1000, CV $<5 \%$ ) particles to the concentration of $\sim 10^{7}$ particles $\mathrm{ml}^{-1}$ by deionized water and PEO solution respectively. This concentration was considered to be low enough to neglect any interaction between particles in the micro-channel. Before the experiment, the particles were shaken by a vortex device to guarantee a good suspension. 


\section{Experimental setup}

The particle suspensions were transferred to a $1 \mathrm{ml}$ syringe, and then introduced into the microfluidic chip through a silicon tube by a syringe pump (Legato 100 , Kd Scientific). The outflow of the particle suspension was collected in a glass bottle. The microfluidic chip was placed on an inverted microscope (CKX41, Olympus, Japan), and illuminated by a mercury arc lamp. The images of the fluorescent particles were observed and captured by a CCD camera (Rolera Bolt, Q-imaging, Australia) which had a maximum capturing speed of 50 frames per second. The fluorescent images were then post-processed and analysed with the software Q-Capture Pro 7 (Q-imaging, Australia). The flow rate in the experiment was increased from $10 \mu \mathrm{Imin}^{-1}$ to $300 \mu \mathrm{min}^{-1}$, which corresponds to an average fluid velocity from $0.04 \mathrm{~m} \mathrm{~s}^{-1}$ to $1.2 \mathrm{~m}$ $\mathrm{s}^{-1}$. A profile of the fluorescent intensity was taken from the outlet of the last cavity to examine the focusing performance of this microfluidic device in both Newtonian and non-Newtonian fluid.

\section{RESULTS AND DISCUSSION}

\section{A. Effects of elasticity on particle migration}

Figure 6 shows the fluorescent images of 3.2- $\mu \mathrm{m}, 4.8-\mu \mathrm{m}, 13-\mu \mathrm{m}$ particles at the outlet under the flow rate of $60 \mu \mathrm{min} \mathrm{m}^{-1}$ in both Newtonian and non-Newtonian fluids, respectively, and corresponding intensity profiles for each particle type along the width of the channel. In the 500-ppm PEO dysolution, three particle types were focused very well at the flow rate of $60 \mu \mathrm{min}^{-1}\left(R c=4.62, W_{i}=45.48, E l=9.84\right)$ and in a specific range of flow rate around $60 \mu \mathrm{min} \mathrm{m}^{-1}$ as well. The particles experience the lift force $F_{L}$, the dean drag force $F_{D}$ and the elastic force $F_{E}$ at the same time, and an equilibrium position was observed on the opposite of cavity side due to the combined effects of the three forces. In a Newtonian fluid, however, no obvious particle focusing is observed at the outlet under $60 \mu \mathrm{min}^{-1}$ flow rate with the 3.2- $\mu \mathrm{m}, 4.8-\mu \mathrm{m}$ particle size. The 13- $\mu \mathrm{m}$ particles begin to focus from $60-\mu \mathrm{l} \mathrm{min}{ }^{-1}$ flow rate under the effects of the lift force $F_{L}$, the dean drag force $F_{D}$. It can also be seen from the fluorescent intensity profile (Figure $6 \mathrm{~d}, \mathrm{e}, \mathrm{f}$ ) that the particles in DI water are mostly dispersed, while in PEO solution the particles are tightly focused. The width of the focusing line (derived from the difference of lateral position in the fluorescence intensity profile at $70 \%$ intensity peak) in PEO solution is similar to the diameter of the particles. It's concluded that single-particle focusing is achieved in the $x-y$ plane. Compared with particle focusing in a Newtonian fluid, the elasticity in a non-Newtonian fluid accelerates the particle focusing, improves the focusing performance, and finally focuses particles on the opposite side of cavity.

(a)

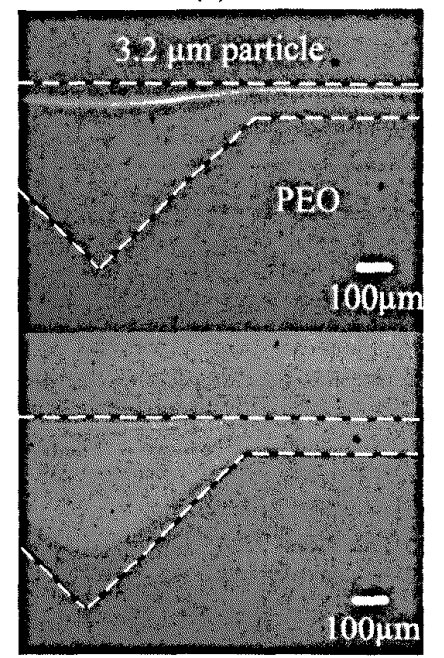

(d)

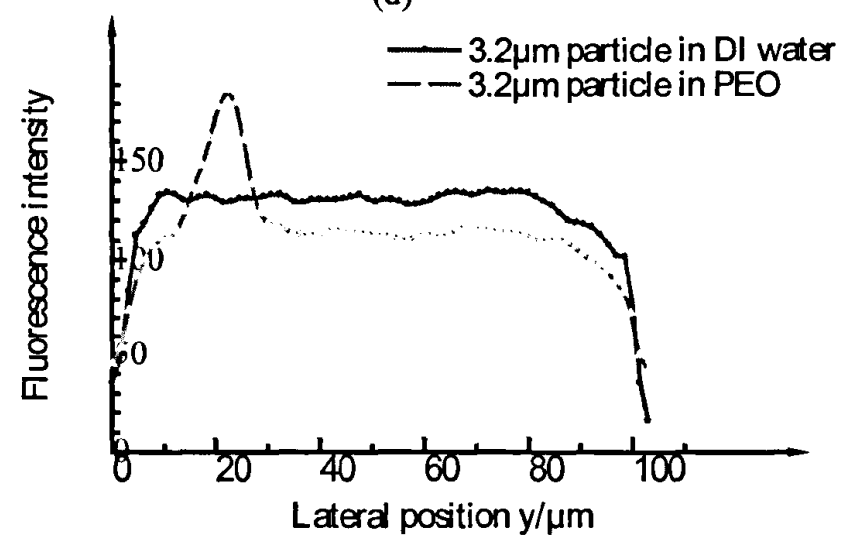


(b)

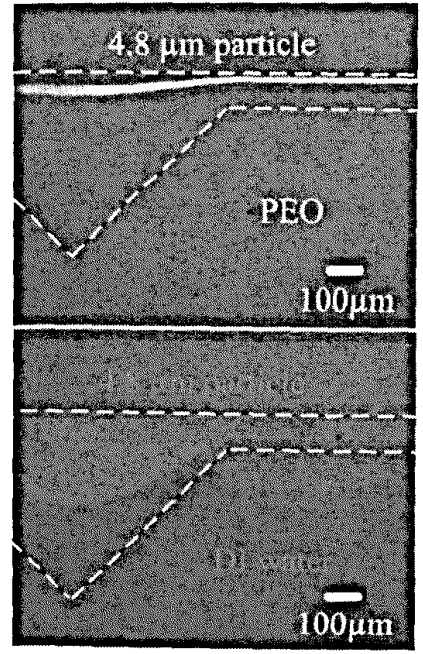

(c)

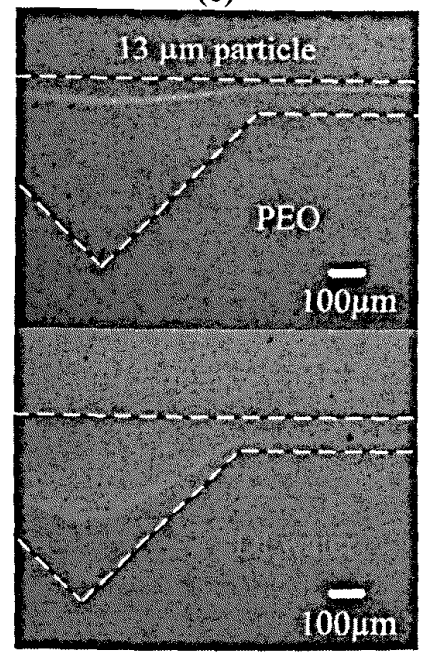

(e)
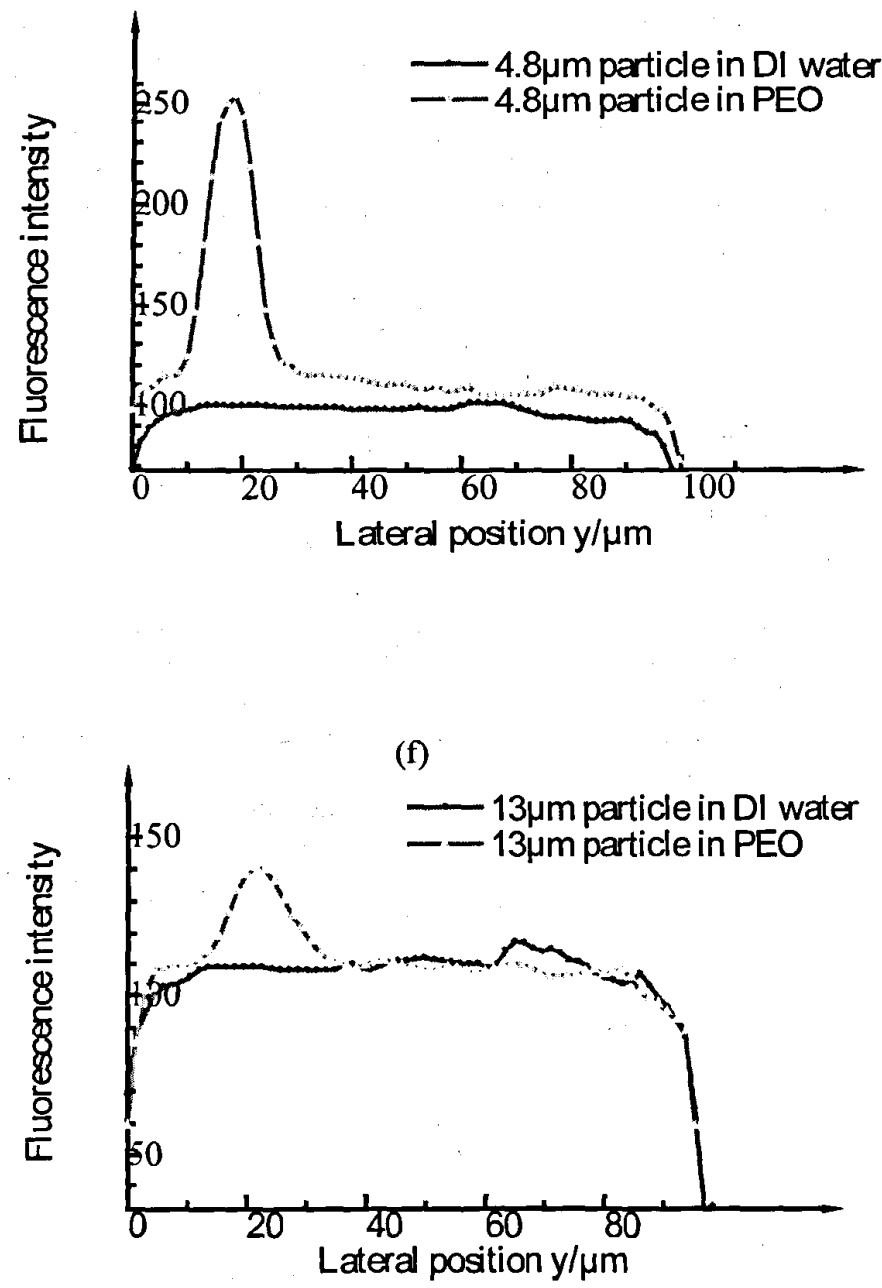

Figure 6 The fluorescent images of $3.2-\mu \mathrm{m}, 4.8-\mu \mathrm{m}, 13-\mu \mathrm{m}$ particles at the outlet under the flow rate of $60 \mu \mathrm{lmin} \mathrm{m}^{-1}$ in Newtonian and non-Newtonian fluids, respectively, and corresponding fluorescent intensity profiles for each particle type along the width of the channel.

\section{B. Effects of different flowing conditions.}

As the inertial lift force, secondary force and elastic force are all related to the flow rate. Under different flowing conditions, the particles will experience different dominant forces, which will lead to different particle distributions. Take 4.8- $\mu \mathrm{m}$ particle as an example, the $4.8-\mu \mathrm{m}$ particles distribution was observed under different flowing conditions in $500 \mathrm{ppm}$ PEO fluid and DI water, as is shown in Figure 7. The flow rate $Q$ varies from $10 \mu 1 \mathrm{~min}^{-1}$ to $300 \mu \mathrm{l} \mathrm{min}{ }^{-1}$, which corresponds to the levels of $R_{c}$ ranging from 0.77 to $23.10, W_{i}$ ranging from 7.58 to 227.4 , and $E l 9.84$ which is independent of the flow rate. In DI water, $W_{i}$ and $E l$ are both zero since Newtonian fluid does not exhibit elastic properties. When the flow rates are relatively low $\left(<30 \mu 1 \mathrm{~min}^{-1}, R_{c}=2.31, W_{i}=22.74, E l=9.84\right)$ with $500 \mathrm{ppm}$ PEO solution, the inertial effect and the Dean effect are negligble, while elastic force is dominant, and the particles are randomly distributed. As the flow rate increases, the inertial and Dean drag force being exerted on a particle competes 
with elastic force, and the particles gradually migrate to the opposite side of the cavity in the channel and focused tightly

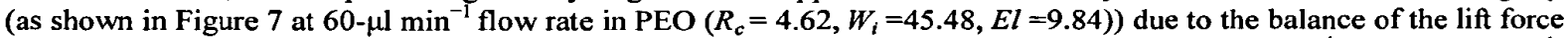
$F_{L}$, Dean drag force $F_{D}$ and elastic force $F_{E}$. The focusing range is approximately from $30 \mu 1 \mathrm{~min}^{-1}$ to $120 \mu \mathrm{min}$. When the flow rate reaches $120 \mu \mathrm{I} \mathrm{min}^{-1}\left(R_{c}=9.24, W_{i}=90.96, E l=9.84\right)$, another focusing line occurs on the cavity side. This is partly because inertia and secondary flow effects begin to dominate the particle migration. Consequently, the elastic force was eventually overwhelmed by the inertial force all the particles migrate and focus on the cavity side when the flow rate reaches $240 \mu \mathrm{lmin}^{-1}\left(R_{c}=18.48, W_{i}=181.92, E l=9.84\right)$. In DI water, the particles are dispersed until the flow rate reaches $120 \mu \mathrm{min}^{-1}$, then the particles are focused on the cavity side of the channel under the combined effect of the lift force $F_{L}$ and the Dean drag force $F_{D}$. The minimum focusing flow rate in a Newtonian fluid is higher than that in a non-Newtonian fluid and the focusing performance in a Newtonian fluid is not as good as in a PEO solution. That is to say, the elastic effect accelerates and improves the particle focusing performance. It can also be seen from the fluorescent intensity profiles of particles flowing with different flow rates in 500ppm PEO and DI water. A further advantage is that in ECCA channel the flow rate that allows particle focusing is dozens of or even hundreds of microlitre per minute, which is much higher than that in a straight channel (several microlitres per minute) ${ }^{21}$, resulting in a higher throughtput $\left(>10000 \mathrm{~s}^{-1}\right)$.
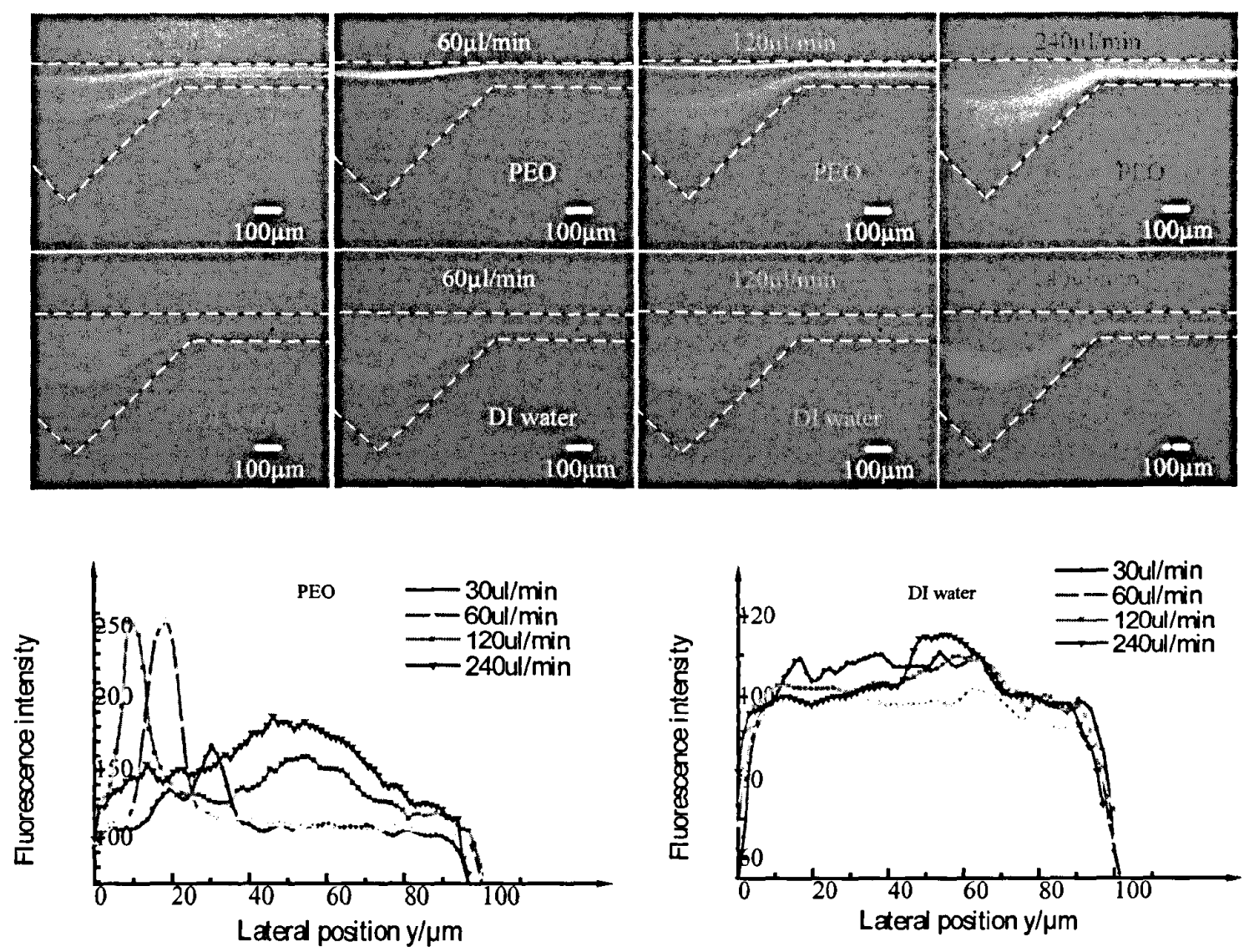

Figure 7 The fluorescent images of $4.8 \mu \mathrm{m}$ particle at the outlet at the flow rate of $30 \mu \mathrm{l} \mathrm{min}, 60 \mu \mathrm{min} \mathrm{mi}^{-1}, 120 \mu \mathrm{lmin}$, $240 \mu 1 \mathrm{~min}^{-1}$ in 500ppm PEO and DI water fluids, respectively, and corresponding fluorescent intensity profiles for different flow rates along the width of the channel.

\section{Side view of the channel}

Figure 8 (a) is the side view of the channel when particles flowing in 500ppm PEO solution at a flow rate of $60 \mu \mathrm{min} \mathrm{m}^{-1}$. It is verified that the particles flowing in non-Newtonian fluid are not only focused in the $x-y$ plane, but also form a single line in the centreline of the channel in the $x-z$ plane. That means, the $3 \mathrm{D}$ particle focusing in non-Newtonian fluid 
in this ECCA channel is realized by properly balancing the effects of inertia, secondary flow and elasticity. This phenomenon was not observed for the case of a Newtonian fluid when particles focused in DI water in $x-y$ plane as shown in Figure 8 (b) in $240 \mu \mathrm{min}^{-1}$. Instead, the particles in DI water are focused in two lines along the two channel walls in the $x-z$ plane due to the synergetic effect of inertia and secondary flow, which is not suitable for practical applications such as one-by-one particle counting or sorting. It is confirmed that the equilibrium positions are reduced to single one by the additional elasticity effect in PEO solution in ECCA channel. It's also obvious from the corresponding fluorescent intensity profiles in PEO and DI water, respectively. In a PEO solution, only one fluorescence intensity peak exists in the middle of the lateral side view, while there are two peaks in DI water.

(a)

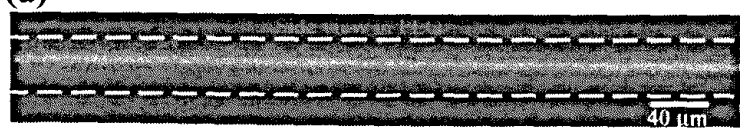

(b)

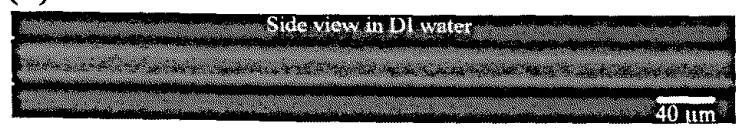

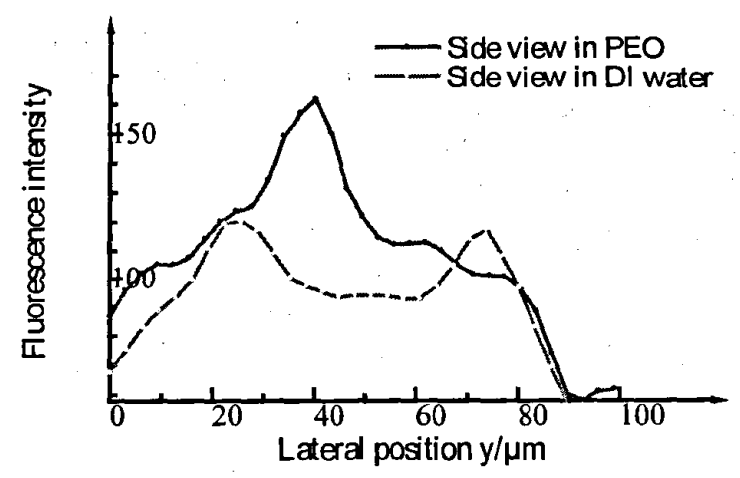

Figure 8 Fluorescent images of the side view of the channel when particle focusing in 500ppm PEO solution and DI water, respectively, and corresponding fluorescent intensity profiles.

\section{CONCLUSION}

To summarize, 3D particle focusing was demonstrated in an ECCA channel by exploiting the Dean-flow-coupled elastoinertial effects. By properly controlling the flow rates to harmonize the inertial force, viscoelastic force and Dean-drag force, 3D particle focusing in non-Newtonian fluid along the opposite side of cavities was achieved. Particle focusing under Newtonian and non-Newtonian fluids using three different sized particles $(3.2 \mu \mathrm{m}, 4.8 \mu \mathrm{m}, 13 \mu \mathrm{m})$ were demonstrated. The particles were focused on the cavity side in a Newtonian fluid by the composition of inertial and Dean-drag force, whereas on the opposite cavity side in a non-Newtonian fluid due to the addition of the viscoelastic force. Compared with particle focusing in Newtonian fluid in an ECCA channel, it can be concluded that particles in non-Newtonian fluid are more easily and better focused. In the ECCA channel, the flow rate that allows particle focusing is dozens of or even hundreds of microlitre per minute, which is much higher than that in a straight channel (several microlitres per minute) ${ }^{21}$, resulting in a higher throughtput $\left(>10000 \mathrm{~s}^{-1}\right)$. This Dean-flow-coupled elasto-inertial microfluidic device could offer a continuous, sheathless, and high-throughput ( $\left.>10000 \mathrm{~s}^{-1}\right)$ 3D focusing performance, which may be valuable in various applications from high speed flow cytometry to cell counting, sorting, and analysis.

\section{ACKNOWLEDGEMENTS}

This work is supported by the University of Wollongong -China Scholarship Council joint scholarships.

\section{REFERENCES}

1 Whitesides, G. M., "The origins and the future of microfluidics," Nature, 442(7101), 368-373 (2006).

2 Bhagat, A. A., Kuntaegowdanahalli, S. S. S., Kaval, Seliskar, N., C. J., and Papautsky, I., "Inertial microfluidics for sheath-less high-throughput flow cytometry," Biomedical microdevices, 12(2), 187-195 (2010).

$3 \mathrm{Kang}, \mathrm{Y}$., Li, D., Kalams, S. A., and Eid, J. E., "DC-Dielectrophoretic separation of biological cells by size," Biomedical microdevices, 10(2), 243-249 (2008). 
$4 \mathrm{Li}$, M., Li, S., Cao, W., Li, W., Wen, W., and Alici, G., "Continuous particle focusing in a waved microchannel using negative dc dielectrophoresis," Journal of Micromechanics and Microengineering, 22(9), 095001 (2012).

5 Yan, S., Zhang, J., Alici, G., Du, H., Zhu, Y., and Li, W., "Isolating plasma from blood using a dielectrophoresis-active hydrophoretic device," Lab on a Chip, 14(16), 2993-3003 (2014).

$6 \mathrm{Kang}, \mathrm{K}$. H., Kang, Y., Xuan, X., and Li, D., "Continuous separation of microparticles by size with Direct currentdielectrophoresis," Electrophoresis, 27(3), 694-702 (2006).

7 Hejazian, M., Li, W. H., Nguyen, N.-T., "Lab on a chip for continuous-flow magnetic cell separation," Lab on a Chip, 15(4), 959-970 (2015).

8 Zeng, J., Chen, C., Vedantam, P., Brown, V., Tzeng, T. R. J., and Xuan, X., "Three-dimensional magnetic focusing of particles and cells in ferrofluid flow through a straight microchannel," Journal of Micromechanics and Microengineering, 22(10), 105018 (2012).

9 Friend, J. and Yeo, L., "Microscale acoustofluidics: Microfluidics driven via acoustics and ultrasonics," Reviews of Modern Physics, 83(2), 647 (2011).

10 MacDonald, M. P., Spalding, G. C. and Dholakia, K, "Microfluidic sorting in an optical lattice," Nature, 426(6965), 421-424 (2003).

11 Karimi, A., Yazdi, S. and Ardekani, A. M., "Hydrodynamic mechanisms of cell and particle trapping in microfluidics," Biomicrofluidics, 7(2), 021501 (2013).

12 Yamada, M., Nakashima, M. and Seki, M., "Pinched flow fractionation: continuous size separation of particles utilizing a laminar flow profile in a pinched microchannel," Analytical chemistry, 76(18), 5465-5471 (2004).

13 Crowley, T. A. and Pizziconi, V., "Isolation of plasma from whole blood using planar microfilters for lab-on-a-chip applications," Lab on a Chip, 5, 922-929 (2005).

14 Di Carlo, D., "Inertial microfluidics," Lab on a Chip, 9(21), 3038-3046 (2009).

15 Zhang, J., Li, M., Li, W. H. and Alici, G., "Inertial focusing in a straight channel with asymmetrical expansioncontraction cavity arrays using two secondary flows," Journal of Micromechanics and Microengineering, 23(8), 085023 (2013).

16 Davis, J. A., Inglis, D. W., Morton, K. J., Lawrence, D. A., Huang, L. R., Chou, S. Y., Sturm, J. C., and Austin, R. H., "Deterministic hydrodynamics: taking blood apart," Proceedings of the National Academy of Sciences, 103(40), 1477914784 (2006).

17 D'Avino, G., Maffettone, P. L., Greco, F. and Hulsen, M. A., "Viscoelasticity-induced migration of a rigid sphere in confined shear flow," Journal of Non-Newtonian Fluid Mechanics, 165(9), 466-474 (2010).

18 Huang, P. Y., Feng, J., Hu, H. H. and Joseph, D. D., "Direct simulation of the motion of solid particles in Couette and Poiseuille flows of viscoelastic fluids," Journal of Fluid Mechanics, 343, 73-94 (1997).

19 Leshansky, A. M., Bransky, A., Korin, N. and Dinnar, U., "Tunable nonlinear viscoelastic "focusing" in a microfluidic device," Physical review letters, 98(23), 234501 (2007).

$20 \mathrm{Ho}$, B. P., and Leal, L. G. "Migration of rigid spheres in a two-dimensional unidirectional shear flow of a secondorder fluid," Journal of Fluid Mechanics, 76(04), 783-799 (1976).

21 Yang, S., Kim, J. Y., Lee, S. J., Lee, S. S., and Kim, J. M., "Sheathless elasto-inertial particle focusing and continuous separation in a straight rectangular microchannel," Lab on a Chip, 11(2), 266-273 (2011).

22 D’Avino, G., Romeo, G., Villone, M. M., Greco, F., Netti, P. A. and Maffettone, P. L., "Single line particle focusing induced by viscoelasticity of the suspending liquid: theory, experiments and simulations to design a micropipe flowfocuser," Lab on a Chip, 12(9), 1638-1645 (2012).

23 Seo, K. W., Byeon, H. J., Huh, H. K., and Lee, S. J., "Particle migration and single-line particle focusing in microscale pipe flow of viscoelastic fluids," RSC Advances, 4(7), 3512-3520 (2014).

24 Asmolov, E. S., "The inertial lift on a spherical particle in a plane Poiseuille flow at large channel Reynolds number," Journal of Fluid Mechanics, 381, 63-87 (1999).

25 Cha, S., Shin, T., Lee, S. S., Shim, W., Lee, G., Lee, S. J., "Cell stretching measurement utilizing viscoelastic particle focusing," Analytical chemistry, vol. 84, 10471-10477 (2012).

26 Xue, S. C., Phan-Thien, N. and Tanner, R, "Three dimensional numerical simulations of viscoelastic flows through planar contractions," Journal of Non-Newtonian Fluid Mechanics, 74, 195-245, (1998).

27 Tehrani, M. A., "An experimental study of particle migration in pipe flow of viscoelastic fluids," Journal of Rheology (1978-present), 40(6), 1057-1077 (1996). 